\title{
DISPERSIVE WAVES AND CAUSTICS
}

\author{
ARTHUR D. GORMAN \\ Department of Engineering Science \\ Lafayette College \\ Easton, Pennsylvania 18042 U.S.A. \\ (Rereived Noverber 30, 1984)
}

ABSTRACT. The Lagrange manifold (WKB) formalism enables the determination of the asymptotic series solution of linear, non-dispersive wave equations at turning points. The formalism is adapted to include those equations which model dispersive waves.

KEYWORDS AND PHRASES. Dispersive waves, Lagrange manifold, turning points, WKB 1980 MATHEMATICS SUBJECT CLASSIFICATION COOE: $34 E 20$

1. INTRODUCTION.

Scalar wave propagation in a spatially inhomogeneous dispersive medium is commonly represented by a partial differential equation of the form

$$
\left.\nabla^{2} \psi(\bar{r}, t)-f(\bar{r}) \frac{\partial^{2} \psi}{\partial t^{2}} \overline{(r}, t\right)-g(\bar{r}) \psi(\bar{r}, t)=0
$$

In Equation (1.1), $\psi(\bar{r}, t)$ is the wave function, $\bar{r}$ refers to the spatial coordinates and $t$ is the time. When associated with propagation in a cold plasma, $f(\bar{r})$ is related to the refractive index and $g(\bar{r})$ to the plasma cyclotron frequency. No general technique exists for solving such equations exactly. Consequently, various approximate solutions, each valid under specific assumptions, are of ten developed. One such approach is the eikonal or geometric optics solution [1]. Although this technique has long been applied to problems involving dispersive waves $[2,3,4]$, recent extensions $[5,6]$ and pedagogical treatments $[7,8]$ continue to emphasize its importance.

The approach applies to dispersive waves when $g(\bar{r})$ varies sufficiently slowly to allow a scaling of coordinates $(\bar{r} \rightarrow \bar{r} / \lambda, t \rightarrow t / \lambda ; \lambda>>0)$ so Equation (1.1) may be written as

$$
\nabla^{2} \psi(\bar{r}, t)-f(\bar{r}) \frac{\partial^{2} \psi}{\partial t^{2}}(\bar{r}, t)-\lambda^{2} g(\bar{r}) \psi(\bar{r}, t)=0
$$

Physically, this corresponds to the regime of long distances and observation times. A solution of the form

$$
\psi(\bar{r}, t)=\exp \{i \lambda S(\bar{r}, t)\} A(\bar{r}, t, \lambda)
$$

where 


$$
A(\bar{r}, t, \lambda) \simeq \sum_{k=0} A_{k}(\bar{r}, t)(i \lambda)^{-k}, \quad A_{-k}=0
$$

is assumed. $S(\bar{r}, t)$ may be regarded as a phase and $A(\bar{r}, t, \lambda)$ as an amplitude. Substituting Equation (1.3) into Equation (1.2) and re-grouping by powers of $1 \lambda$ leads to

$$
\begin{aligned}
& \left\{(i \lambda)^{2}\left[(\nabla S)^{2}-f(\bar{r})\left(\frac{\partial S}{\partial t}\right)^{2}+g(\bar{r})\right]+\right. \\
& i \lambda\left[\nabla^{2} S-f(\bar{r}) \frac{\partial^{2} S}{\partial t^{2}}+2 \nabla S \cdot \nabla-2 f(\bar{r}) \frac{\partial S}{\partial t} \frac{\partial}{\partial t}\right]+ \\
& \left.\left.(i \lambda) 0\left[\nabla^{2}-f(\bar{r}) \frac{\partial^{2}}{\partial t^{2}}\right]\right\} \sum_{k=0} A_{k}(\bar{r}, t)\right)(i \lambda)^{-k} \simeq 0 .
\end{aligned}
$$

Following the usual convention, by introducing the wavenumber and frequency,

$$
\bar{p}=\nabla S \quad \omega=-\frac{\partial S}{\partial t},
$$

respectively, a dispersion (the eikonal) equation is obtained from the coefficient of the $(i \lambda)^{2}$ term, namely

$$
\bar{p} \cdot \bar{p}-f(\bar{r}) \omega^{2}+g(\bar{r})=0 .
$$

If we regard the wavevectors as momenta, Equation (1.7) may be seen as a Hamiltonian

$$
H=\bar{p} \cdot \bar{p}-f(\bar{r}) \omega^{2}+g(\bar{r}) .
$$

Then Hamilton's Equations

$$
\begin{array}{ll}
\frac{d \bar{r}}{d \gamma}=\nabla_{p} H & \frac{d \bar{p}}{d \gamma}=-\nabla_{r} H \\
\frac{d t}{d \gamma}=-\frac{\partial H}{\partial \omega} & \frac{d \omega}{d \gamma}=\frac{\partial H}{\partial t}
\end{array}
$$

may be used to find the trajectories (map)

$$
\begin{array}{ll}
\bar{r}=\bar{r}(\gamma, \bar{\sigma}) & \bar{p}=\bar{p}(\gamma, \bar{\sigma}) \\
t=t(\gamma, \bar{\sigma}) & \omega=\omega(\gamma, \bar{\sigma})
\end{array}
$$

where $\gamma$ is a ray-path parameter and $\bar{\sigma}$ a parameterized initial condition. The sign differences between Equations (1.8) and (1.9) are due to the convention employed in defining $\omega[2]$. (We note that since the Hamiltonian is time independent, the system is conservative and $\frac{d \omega}{d \gamma}=0$.) Integrating along the trajectories obtains the phase

$$
S(\bar{r}, t)=\int_{\bar{r}_{0}, t}^{\bar{r}, t}(\bar{p} \cdot d \bar{r}-\omega d t)+S\left(\bar{r}_{0}, t_{0}\right) .
$$

The amplitudes can now be determined from the coeffictents of the $(i \lambda)$ and $(i \lambda)^{\circ}$ terms in Equation (1.5). Usually these terms are re-grouped, using Equations (1.6), into a first order (transport) equation

$$
\left[\nabla \cdot \bar{p}+f(\bar{r}) \frac{\partial \omega}{\partial t}+2\left(\bar{p} \cdot \nabla+2 \omega f(\bar{r}) \frac{\partial}{\partial t}\right)\right] A_{k}=-\left(\nabla^{2}-f(\bar{r}) \frac{\partial^{2}}{\partial t^{2}}\right) A_{k-1}, k>1
$$

which obtains the $\mathrm{A}_{k}$ 's recursively. 
This algorithm suffices to determine asymptotic solutions at most field points. On caustic curves, regions where the inhomogeneity of the media effects a focusing of trajectories, the map from $(\gamma, \bar{\sigma}) \rightarrow(\bar{r}, t)$ becomes singular, i.e.,

$$
\operatorname{det}\left(\frac{\partial(\bar{r}, t)}{\partial(\gamma, \bar{\sigma})}\right)=0
$$

and the technique predicts unbounded field amplitudes. Such difficulties can often be circumvented using the Lagrange manifold formalism of Maslov [9] and Arnold [10]. A modification of their technique has been applied to the reduced Helmholtz equation [11], enabling straightforward calculation of the field at caustic points while retaining the framework of their formalism $[12,13]$. Here we adapt this modified technique to dispersive waves and extend the approach to more complex caustic geometries than in [11]. Some field aspects specific to electromagnetic wave propagation are also considered.

\section{PRELIMINARIES}

The Lagrange manifold formalism is a mixed coordinate-momentum space approach which leads to finite wave amplitudes on caustic curves. Applied to wave propagation modeled by the reduced Helmholtz equation, the technique involves re-parametrization of the position coordinates (trajectories) in terms of the momenta (wavevectors). This re-parametrization is essentially a level-preserving coordinate transformation, i.e., regular, or non-caustic, points are carried to regular points and singular, or caustic, points are carried to singular points. For example, let waves propagating from a point source at the origin be modeled by the reduced Helmholtz equation

$$
\frac{\partial^{2} \psi}{\partial x^{2}} \quad \frac{\partial^{2} \psi}{\partial y^{2}}+\lambda^{2}(1-x) \psi=0
$$

Near turning (caustic) points of the highest order, we assume an asymptotic solution of the form

$$
\psi(\bar{r})-\int A(\bar{r}, \bar{p}, \lambda) \exp \{i \lambda(\bar{r} \cdot \bar{p}-s(\bar{p}))\} d \bar{p}=0\left(\lambda^{-\infty}\right)
$$

where $A(\bar{r}, \bar{p}, \lambda)$ and its derivatives are bounded, $\bar{r}=(x, y), \bar{p}=\left(p_{x}, p_{y}\right), \lambda$ is a large parameter and the stationary phase condition $\left[\nabla_{p}(\bar{r} \cdot \bar{p}-S(\bar{p}))=0\right]$ determines the Lagrange manifold of Maslov near the turning point. The differentiation in Equation (2.1) is carried across the integral in Equation (2.2) and the terms are re-grouped by powers of $i \lambda$. For clarity, we retain only the $(i \lambda)^{2}$ term

$$
\int(i \lambda)^{2}\left(p_{x}{ }^{2}+p_{y}{ }^{2}+x-1\right) A(\bar{r}, \bar{p}, \lambda) \exp \{i \lambda(\bar{r} \cdot \bar{p}-s(\bar{p}))\} d \bar{p},
$$

from which we obtain Maslov's Hamiltonian

$$
H=p_{x}^{2}+p_{y}^{2}+x-1 \text {. }
$$

Then by invoking the stationary phase condition Maslov's Hamiltonian becomes an 
eikonal on the Lagrange manifold

$$
p_{x}^{2}+p_{y}^{2}+x-1=0
$$

Hamilton's equations lead to the maps

$$
\begin{array}{ll}
x=-t^{2}+2 t \sin \theta & p_{x}=-t+\sin \theta \\
y=2 t \cos \theta & p_{y}=\cos \theta
\end{array}
$$

where here $\theta$ is an initial propagation direction with respect to the y-axis. Inverting the $(t, \theta) \rightarrow\left(p_{x}, p_{y}\right)$ map and substituting into the coordinate space map leads to the Lagrange manifold and phase

$$
\begin{aligned}
& x=1-p_{x}{ }^{2}-p_{y}{ }^{2}=\frac{\partial S}{\partial p_{x}} \quad y=2 p_{y}\left(1-p_{y}{ }^{2}\right)^{1 / 2}-2 p_{x} p_{y}=\frac{\partial S}{\partial p_{y}} \\
& \phi(\bar{r}, \bar{p})=\bar{r} \cdot \bar{p}-s(\bar{p})=x p_{x}+y p_{y}-p_{x}+\frac{1}{3} p_{x}{ }^{3}+p_{x} p_{y}{ }^{2}+\frac{2}{3}\left(1-p_{y}\right)^{3 / 2} .
\end{aligned}
$$

At $(t, \theta)=\left(2,30^{\circ}\right),(x, y)=(-2,3.46)$; the map $(t, \theta) \rightarrow(x, y)$ becomes singular, cf. Equation (1.14). At the corresponding values of the momenta $\left(p_{x}, p_{y}\right)=(-1.5, .866)$, the Hessian determinant of the phase is also zero,

$$
\operatorname{det}\left(\frac{\partial^{2} \phi}{\partial p_{x} \partial p_{y}}\right)=0 \text {. }
$$

Analogously, at points where the $(t, \theta) \rightarrow(x, y)$ map is non-singular, the Hessian determinant above is non-zero. This correspondence between the Jacobian of the map and the Hessian of the phase is central to the formalism.

In applying this technique to dispersive wave propagation, Equation (1.2), a similar level-preserving transformation must be constructed. A disadvantage (compared to the case above) in constructing this coordinate transformation arises from the definition of $\omega$. For, just as in the classical approach, where the conventional definition of $\omega$ led to a sign difference in Hamilton's space-time equations $[2,3,4]$, the sign differences involved in the conventional definitions of $\bar{p}$ and $\omega$, Equations (1.6), must be accounted for here. Because the explicit form of the phase is so crucial to the formalism, one approach to accounting for this sign difference is in the phase. Specifically, rather than assuming an asymptotic solution involving the usual form $\exp \{i \lambda(\bar{r} \cdot \bar{p}-\omega t)\}$, we assume Equation (1.2) has an asymptotic solution of the form

$$
\psi(\bar{r})-\int A(\bar{r}, \tau, \bar{p}, \omega, \lambda) \exp \{i \lambda(\bar{r} \cdot \bar{p}+\omega \tau-S(\bar{p}, \omega))\} d \bar{p} d \omega=0\left(\lambda^{-\infty}\right)
$$

where $\tau$ is a function of time, namely

$$
\tau=-t \text {. }
$$

Since Equation (1.2) is time-symmetric, its form remains the same under this substitution. For causality, we shall consider $\tau<0$, which corresponds to $t>0$ in the classical development above, and define the phase as

$$
\phi(\bar{r}, \tau, \bar{p}, \omega)=\bar{r} \cdot \bar{p}+\omega \tau-s(\bar{p}, \omega) .
$$


Using this device allows the straightforward construction of the required levelpreserving coordinate transformation while retaining the classical definition of $\omega$

$$
\omega=\frac{\partial \phi}{\partial \tau}=-\frac{\partial \phi}{\partial t},
$$

as well as the form of Hamilton's equations given by Weinberg [2].

3. FORMALISM

To begin the algorithm, we first introduce $\tau$ into Equation (1.2) obtaining

$$
\nabla^{2} \psi-f(\bar{r}) \frac{\partial^{2} \psi}{\partial \tau^{2}}-\lambda^{2} g(\bar{r}) \psi=0
$$

and assume the asymptotic solution from above

$$
\psi(\bar{r})-\int A(\bar{r}, \tau, \bar{p}, \omega, \lambda) \exp \{i \lambda(\bar{r} \cdot \bar{p}+\omega \tau-S(\bar{p}, \omega))\} d \bar{p} d \omega=0\left(\lambda^{-\infty}\right) .
$$

The technique proceeds by carrying the differentiation in Equation (3.1) across the integral in Equation (2.5) resulting in

$$
\begin{aligned}
& \int d \bar{p} d \omega \exp \{i \lambda(\bar{r} \cdot \bar{p}+\omega \tau-s(\bar{p}, \omega))\}\left\{(i \lambda)^{2}\left(\bar{p} \cdot \bar{p}-f(\bar{r}) \omega^{2}+g(\bar{r})\right) A+\right. \\
& \left.(i \lambda)\left(2 \bar{p} \cdot \nabla A-2 \omega f(\bar{r}) \frac{\partial A}{\partial \tau}\right)+(i \lambda)^{0}\left(\nabla^{2} A-f(\bar{r}) \frac{\partial^{2} A}{\partial \tau^{2}}\right)\right\}=0\left(\lambda^{-\infty}\right) .
\end{aligned}
$$

Maslov's Hamiltonian is the coefficient of the $(i \lambda)^{2}$ term

$$
H=\bar{p} \cdot \bar{p}-f(\bar{r}) \omega^{2}+g(\bar{r}) .
$$

Then by invoking the stationary phase condition

$$
\nabla_{q} \phi=\left(\hat{p} \cdot \nabla_{p}+\hat{\omega} \frac{\partial}{\partial \omega}\right) \phi=0
$$

which defines the Lagrange manifold

$$
\bar{r}=\nabla_{p} p \quad \tau=\frac{\partial \phi}{\partial \omega},
$$

Maslov's Hamiltonian becomes an eikonal equation on the Lagrange manifold

$$
\bar{p} \cdot \bar{p}-f\left(\nabla_{p} s\right) \omega^{2}+g\left(\nabla_{p} s\right)=0 \text {, }
$$

cf. Equation (1.7). To obtain the phase, we first use Hamilton's Equations

$$
\begin{array}{ll}
\frac{d \bar{r}}{d \gamma}=\nabla_{p} H & \frac{d \bar{p}}{d \gamma}=-\nabla_{r} H \\
\frac{d \tau}{d \gamma}=\frac{\partial H}{\partial \omega} & \frac{d \omega}{d \gamma}=-\frac{\partial H}{\partial \tau},
\end{array}
$$

noting the equivalence of Equations (3.8) and (1.9) using $t=-\tau$, to find the trajectories

$$
\begin{array}{ll}
\bar{r}=\bar{r}(\gamma, \bar{\sigma}) & \bar{p}=\bar{p}(\gamma, \bar{\sigma}) \\
\tau=\tau(\gamma, \bar{\sigma}) & \omega=\omega(\gamma, \bar{\sigma}),
\end{array}
$$

where $\gamma$ is the ray-path parameter and $\bar{\sigma}$ a set of parametrized initial conditions, e.g., direction cosines. Then inverting the $(\gamma, \bar{\sigma}) \rightarrow(\bar{p}, \omega)$ map obtains

$$
\gamma=\gamma(\bar{p}, \omega) \quad \bar{\sigma}=\bar{\sigma}(\bar{p}, \omega) .
$$


Next, substituting into the $(\bar{r}, \tau)$ equations explicitly determines the Lagrange manifold

$$
\begin{aligned}
\bar{r} & =\bar{r}(\gamma(\bar{p}, \omega), \bar{\sigma}(\bar{p}, \omega))=\nabla_{p} S(\bar{p}, \omega) \\
\tau & =\tau(\gamma(\bar{p}, \omega), \bar{\sigma}(\bar{p}, \omega))=\frac{\partial}{\partial \omega} S(\bar{p}, \omega) .
\end{aligned}
$$

Finally, by integrating along the trajectories in Equations (3.12) and (3.13) we obtain

$$
S(\bar{p}, \omega)=\int_{\bar{p}_{0}, \omega_{0}}^{\bar{p}, \omega}(\bar{r} \cdot d \bar{p}+\tau d \omega)+S\left(\bar{p}_{0}, \omega_{0}\right)
$$

analogous to Equation (1.12), and thus the phase

$$
\phi(\bar{r}, \tau, \bar{p}, \omega)=\bar{r} \cdot \bar{p}+\omega \tau-S(\bar{p}, \omega) .
$$

Just as in Equation (2.4), setting the Hessian determinant of the phase to zero

$$
\operatorname{det}\left\{\frac{\partial^{2} \phi}{\partial(\bar{p}, \omega)}\right\}=0 \text {, }
$$

determines the caustic curve in $(\bar{p}, \omega)$ - space. Each point $\left(\bar{p}_{c}, \omega_{c}\right)$ on this curve corresponds to a space-time $\left(\bar{r}_{c}, t_{c}\right)$ point which is found by explicit substitution in the Lagrange manifold and using $t_{c}=-\tau_{c}$ (Equation (2.6))

$$
\begin{aligned}
& \bar{r}_{c}=\nabla_{p} S\left(\bar{p}_{c}, \omega_{c}\right) \\
& \tau_{c}=-\tau_{c}=-\frac{\partial S}{\partial \omega}\left(\bar{p}_{c}, \omega_{c}\right) .
\end{aligned}
$$

The locus of these points specifies the caustic in $(\bar{r}, t)$-space.

To obtain a transport equation for the amplitudes, the Hamiltonian is Taylor expanded near the Lagrange manifold

$$
\bar{p} \cdot \bar{p}-f(\bar{r}) \omega^{2}+g(\bar{r})=\bar{p} \cdot \bar{p}-f\left(\nabla_{p} S\right) \omega^{2}+g\left(\nabla_{p} S\right)+\left(\bar{r}-\nabla_{p} S\right) \cdot \bar{D}_{r}+\left(\tau-\frac{\partial S}{\partial \omega}\right) D_{\tau}
$$

where

$$
\left.\bar{D}_{r}=\int_{0}^{1} \nabla_{r} H\left(\mu\left(\bar{r}-\nabla_{p} S\right)+\nabla_{p} S, \frac{\partial S}{\partial \omega}\right) d \mu \quad D_{\tau}=\int_{0}^{1} \frac{\partial}{\partial \tau} H\left(\nabla_{p} S, \mu\left(\tau-\frac{\partial S}{\partial \omega}\right)+\frac{\partial S}{\partial \omega}\right)\right) d \mu,
$$

i.e., the remainder terms in the Taylor series less factors of $\left(\bar{r}-\nabla_{p} S\right)$ and $\left(\tau-\frac{\partial S}{\partial \omega}\right)$, respectively. By substituting Equation (3.17) into Equation (3.2), noting

$$
\int d \bar{p} d \omega \nabla_{q} \cdot[A \bar{D} \exp \{1 \lambda(\bar{r} \cdot \bar{p}+\omega \tau-s(\bar{p}, \omega))\}]=
$$

$\int d \bar{p} d \omega \exp \{i \lambda(\bar{r} \cdot \bar{p}+\omega \tau-S(\bar{p}, \omega))\}\left\{i \lambda A\left(\bar{r}-\nabla_{p} S\right) \cdot \bar{D}_{r}+i \lambda A\left(\tau-\frac{\partial S}{\partial \omega}\right) D_{\tau}+\bar{D} \cdot \nabla_{q} A+A \nabla_{q} \cdot \bar{D}\right\}$,

where $\bar{D}=\left(\bar{D}_{r}, D_{\tau}\right)$, and taking the surface integral over a sufficiently large radius that it vanishes, Equation (3.2) becomes 


$$
\begin{aligned}
\int d \bar{p} d \omega \exp \{i \lambda(\bar{r} \cdot \bar{p}+\omega \tau-s(\bar{p}, \omega))\}\left\{-i \lambda \nabla_{q} A \cdot \bar{D}-1 \lambda A \nabla_{q} \cdot \bar{D}+\right. \\
\left.2 i \lambda\left(\bar{p} \cdot \nabla_{r} A-f(\bar{r}) \omega \frac{\partial A}{\partial \tau}\right)+\left(\nabla_{r}{ }^{2} A-f(\bar{r}) \frac{\partial^{2} A}{\partial \tau^{2}}\right)\right\}=0\left(\lambda^{-\infty}\right) .
\end{aligned}
$$

Then requiring

$$
-\nabla_{q} A \cdot \bar{D}-A \nabla_{q} \cdot \bar{D}+2 \bar{p} \cdot \nabla_{r} A-2 f(r) \omega \frac{\partial A}{\partial \tau}+\frac{1}{i \lambda}\left(\nabla_{r}{ }^{2} A-f(\bar{r}) \frac{\partial^{2} A}{\partial \tau^{2}}\right)=0
$$

in a neighborhood of the Lagrange manifold leads to a transport equation if we introduce the flow

$$
\begin{array}{ll}
\frac{d \bar{r}}{d \gamma}=2 \bar{p} & \frac{d \bar{p}}{d \gamma}=-\bar{D}_{r} \\
\frac{d \tau}{d \gamma}=-2 f(\bar{r}) \omega & \frac{d \omega}{d \gamma}=-D_{\tau} .
\end{array}
$$

That is, Equation (3.20) is satisfied, and consequently Equation (2.5) is an asymptotic solution, if we allow the asymptotic series

$$
A(\bar{r}, \tau, \bar{p}, \omega, \lambda)=\sum_{k=0} A_{k}(\bar{r}, \tau, \bar{p}, \omega)(i \lambda)^{-k}
$$

to evolve along the transport equation

$$
\frac{d A}{d \gamma}-A \nabla_{q} \cdot \bar{D}+\frac{1}{i \lambda}\left(\nabla_{r}^{2} A-f(\bar{r}) \frac{\partial^{2} A}{\partial \tau^{2}}\right)=0 .
$$

The determination of the asymptotic series of the integrals

$$
\int A_{k}(\bar{r}, \tau, \bar{p}, \omega) \exp \{i \lambda \phi(\bar{r}, \tau, \bar{p}, \omega)\} \overline{d p} d \omega
$$

where $\phi(\bar{r}, \tau, \bar{p}, \omega)$ and $A_{k}(\bar{r}, \tau, \bar{p}, \omega)$ follow from Equations (3.15) and (3.22) respectively, usually proceeds by transforming the phase to its canonical form. Off the caustic (non-zero Hessian determinant) or if the Hessian matrix has one zero eigenvalue, this form is

$$
\tilde{\bar{\phi}}\left(\bar{r}_{0}, \tau, \bar{\beta}\right)=\phi\left(\bar{r}_{0}, \tau_{0}, \bar{p}_{0}, \omega_{0}\right) \pm \beta_{1}{ }^{2} \pm \beta_{2}{ }^{2} \pm \beta_{3}{ }^{2} \pm \beta_{4}{ }^{n} .
$$

If the field point is not a caustic point, $n=2$; the sign of each $\beta_{i}$ is that of the corresponding eigenvalue and the classical stationary phase technique applies to the transformed integral. If the field point is a caustic point, the value of $\mathrm{n}$ is determined from the relative degeneracy of the Hessian based on a criterion derived from a Theorem of Thom (Appendix).

Several approaches may be used to construct the coordinate transformations that carry the phase to its canonical form. If $n=2$, a Taylor expansion of the phase followed by completing the square (alternatively, a principal axis transformation and a re-grouping) obtains the required form and the coordinate transformations. If $n>2$, a Taylor expansion of the phase followed by a princtpal axis transformation obtains 


$$
\begin{gathered}
\left.\simeq \bar{r}_{0}, \tau_{0}, \xi\right)=\phi\left(\bar{r}_{0}, \tau_{0}, \bar{p}_{0}, \omega_{0}\right)+\sum_{i=1}^{3} \lambda_{i} \xi_{i}^{2}+\sum_{i=0} k_{i} \xi_{4}^{n+1}+ \\
\sum_{i j k} c_{i j k} \xi_{i} \xi_{j} \xi_{k}+\text { higher order terms, }
\end{gathered}
$$

where the $\lambda_{i}$ are eigenvalues and the stationary point has been translated to the origin for clarity. If Equation (3.24) cannot be transformed to Equation (3.23) using algebra alone, either of two other basic approaches may be used. One procedure involves using the Cauchy inversion theorem to make explicit the algorithm in the Splitting Lemma [14]. As this construction has been detailed in a similar context [11], we sketch instead the other procedure, a straightforward Taylor series approach $[15,16]$ which extends to the case where the Hessian matrix has two zero eigenvalues. Specifically, following Gilmore [16], we let

$$
\begin{aligned}
& \left.\beta_{i}=\left.\frac{1}{\mid \lambda_{i}}\right|^{\left(\xi_{1}\right.}+\sum_{\alpha+n+\sigma+\mu>2} A^{i}{ }_{\alpha n \delta \mu} \xi_{1}{ }^{\alpha} \xi_{2}{ }^{n} \xi_{3}{ }^{\delta} \xi_{4}{ }^{\mu}\right) i=1,2,3 \\
& \beta_{4}=\xi_{4}\left(\underset{j=0}{=} k_{j} \xi_{4}^{j}\right)^{1 / n} \text {. }
\end{aligned}
$$

Then substituting the above into Equation (3.23), expanding and comparing termwise with Equation (3.24) leads to algebraic equations for the $A_{\alpha n \delta \mu}$ and $k_{j}$, specifying the transformation.

If the Hessian matrix of $p\left(\bar{r}_{0}, \tau_{0}, \bar{p}_{0}, \omega_{0}\right)$ has two zero eigenvalues, the canonical form of the phase is

$$
\left.\overline{\phi\left(\bar{r}_{0}, \tau\right.}, \bar{\beta}\right)=\phi\left(\bar{r}_{0}, \tau_{0}, \bar{p}_{0}, \omega_{0}\right) \pm \beta_{1}^{2} \pm \beta_{2}{ }^{2}+U\left(\beta_{3}, \beta_{4}\right),
$$

where $U\left(B_{3}, B_{4}\right)$ is the appropriate Thom umbilic specified by the criterion in the Appendix. While an extension of the algorithm based on the Splitting Lemma may be used to construct the coordinate transformations carrying Equation ( 3.24 ) to the form above, the Taylor series approach is more straightforward. For brevity, we consider only the case involving the eliptic umbilic. Similar treatmen:s apply to the hyperbolic and parabolic umbilics.

First, the phase is Taylor expanded and after a principal axis transformation is re-grouped in the form

$$
\begin{aligned}
& \simeq \overline{\left(\bar{r}_{0}, \tau_{0}, \bar{\xi}\right)}=\phi\left(\bar{r}_{0}, \tau_{0}, \bar{p}_{0}, \omega_{0}\right)+\sum_{1=1} \lambda_{i} \xi_{1}{ }^{2}+ \\
& \kappa_{30} \xi_{3}{ }^{3}+\kappa_{21} \xi_{3}{ }^{2} \xi_{4}+\kappa_{12} \xi_{3} \xi_{4}{ }^{2}+\kappa_{03} \xi_{4}{ }^{3}+h_{1}\left(\xi_{3}, \xi_{4}\right)+h_{2}\left(\xi_{1}, \xi_{2}, \xi_{3}, \xi_{4}\right),
\end{aligned}
$$

where $h_{1}\left(\xi_{3}, \xi_{4}\right)$ consists of higher order terms in $\xi_{3}$ and $\xi_{4}$ alone and $h_{2}\left(\xi_{1}, \xi_{2}, \xi_{3}, \xi_{4}\right)$ consists of the remaining terms. To carry the cubic terms in Equation (3.27) to the form of the elliptic umbilic

$$
U_{E}\left(\gamma_{3}, \gamma_{4}\right)=\gamma_{3}{ }^{2} \gamma_{4}-\gamma_{4}^{3},
$$

first a linear transformation is introduced

$$
\gamma_{i}=\xi_{1} \quad i=1,2
$$




$$
\left[\begin{array}{c}
r_{3} \\
r_{4}
\end{array}\right]=\left[\begin{array}{ll}
\rho_{11} & \rho_{12} \\
\rho_{21} & \rho_{22}
\end{array}\right]\left[\begin{array}{l}
\xi_{3} \\
\xi_{4}
\end{array}\right]
$$

into Equation (3.27). Comparing the cubic terms in $\gamma_{3}$ and $\gamma_{4}$ which result with Equation (3.28) determines the $\rho_{i j}$ and puts Equation (3.27) into the form $\left.\simeq \overline{(\bar{r}}, \tau_{0}, \bar{\gamma}\right)=\phi\left(\bar{r}_{0}, \tau_{0}, \bar{p}_{0}, \omega_{0}\right)+\sum_{i=1}^{2} \lambda_{i} \gamma_{i}{ }^{2}+\gamma_{3}{ }^{2} \gamma_{4}-\gamma_{4}{ }^{3}+\hat{h}_{1}\left(\gamma_{3}, \gamma_{4}\right)+\hat{h}_{2}\left(\gamma_{1}, \gamma_{2}, \gamma_{3}, \gamma_{4}\right)$

where $\hat{h}_{1}$ and $\hat{h}_{2}$ are the transformed $h_{1}$ and $h_{2}$, respectively. To remove the higher order terms, and thus obtain the canonical form (Equation (3.26)), we proceed as above and insert the transformations

$$
\begin{array}{ll}
B_{i}=\gamma_{i}+\sum_{\alpha+n+\delta+\mu>2}{ }^{i}{ }_{\alpha, \delta} \delta \gamma_{1}{ }^{\alpha} \gamma_{2}{ }^{n} \gamma_{3}{ }^{\delta} \gamma_{4} \mu & i=1,2 \\
\beta_{i}=\gamma_{i}+\sum_{m+n>2} B_{m n}{ }^{i} \gamma_{3}^{m} \gamma_{4} n & i=3,4
\end{array}
$$

into Equation (3.26). Then expanding and making a term-by-term comparison with Equation (3.29) leads to equations for the coefficients in Equation (3.30) and thus specifies the transformations carrying the phase to its canonical form [16]. Similar treatments apply to cases involving the other umbilics.

After the appropriate coordinate transformation, the resulting integral at any field point $\left(\bar{r}_{0}, \tau_{0}\right)$ is of the form

$$
\psi(\bar{r}, \tau) \simeq \int d \bar{B} \exp \left\{i \lambda \tilde{\phi}\left(\bar{r}_{0}, \tau_{0}, \bar{B}\right)\right\} \tilde{\bar{A}}\left(\bar{r}_{0}, \tau_{0}, \bar{B}, \lambda\right)
$$

where

$$
\tilde{\bar{A}}\left(\overline{\mathbf{r}}_{0}, \tau_{0}, \bar{\beta}, \lambda\right)=\mathbf{A}\left(\overline{\mathbf{r}}_{0}, \tau_{0}, \bar{p}(\bar{\beta}), \omega(\bar{\beta}), \lambda\right) \frac{\partial\left(\mathrm{p}_{\mathbf{x}}, \mathrm{p}_{\mathbf{y}}, \mathrm{p}_{z}, \omega\right)}{\partial\left(\beta_{1}, \beta_{2}, \beta_{3}, \beta_{4}\right)}
$$

and the phase is one of

$$
\begin{aligned}
& \tilde{\phi}\left(\bar{r}_{0}, \tau_{0}, \bar{\beta}\right)=\phi\left(\bar{r}_{0}, \tau_{0}, \bar{p}_{0}, \omega_{0}\right) \pm B_{1}{ }^{2} \pm B_{2}{ }^{2} \pm B_{3}{ }^{2} \pm B_{4}{ }^{n} \\
& \tilde{\bar{\phi}}\left(\bar{r}_{0}, \tau_{0}, \bar{B}\right)=\phi\left(\bar{r}_{0}, \tau_{0}, \bar{p}_{0}, \omega_{0}\right) \pm B_{1}{ }^{2} \pm B_{2}{ }^{2} \pm U\left(B_{3}, \beta_{4}\right),
\end{aligned}
$$

where $U\left(\beta_{3}, \beta_{4}\right)$ is an umbilic. If the phase is of the form in Equation (3.23) with $n=2$, the classical stationary phase technique applies directly. If the phase is of the form in Equation (3.23) with $n>2$, Equation (3.31) is regrouped into $\exp \left\{i \lambda \phi\left(\bar{r}_{0}, \tau_{0}, \bar{p}_{0}, \omega_{0}\right)\right\} \int d \beta_{4} \exp \left\{ \pm i \lambda \beta_{4}{ }^{n}\right\} \int d \beta_{1} d \beta_{2} d \beta_{3} \exp \left\{i \lambda\left( \pm \beta_{1}{ }^{2} \pm \beta_{2}{ }^{2} \pm \beta_{3}{ }^{2}\right)\right\} \tilde{\tilde{A}}\left(\bar{r}_{0}, \tau, \bar{B}, \lambda\right)$.

The classical stationary phase technique applies to the multiple integral. Each term in the classical expansion is multiplied by the integral over $B_{4}$ to which a modified stationary phase technique applies [11], resulting in the full expansion of the integral in Equation (3.31).

If the phase in Equation (3.31) is of the form in Equation (3.26), analogous to 
the procedure above, the integral is re-grouping into $\exp \left\{i \lambda \phi\left(\bar{r}_{0}, \tau_{0}, \bar{p}_{0}, \omega_{0}\right)\right\} \int d B_{3} d \beta_{4} \exp \left\{i \lambda U\left(\beta_{3}, \beta_{4}\right)\right\} \int d \beta_{1} d \beta_{2} \exp \left\{i \lambda\left( \pm B_{1}{ }^{2} \pm B_{2}{ }^{2}\right)\right\} \tilde{\bar{A}}\left(\bar{r}_{0}, \tau, \bar{B}, \lambda\right)$. The classical stationary phase technique applies to the integration over $\beta_{1}$ and $\beta_{2}$. Each term in this expansion is multiplied by the integral over $B_{3}$ and $B_{4}$, to which a modified stationary phase technique applies $[17,18]$, resulting in the full expansion. 4. EXAMPLE

Although the formalism is relatively straightforward, we choose an example in $(x, y, t)$ space to reduce the cumbersome algebra. To enable easier comparison with the example illustrating the algorithm that applies to non-dispersive waves [11], we consider a related example with normalized units. Specifically, we consider waves propagating from a point source located at the origin $(x, y)=(0,0)$ at time $t=0$ in a medium described by Equation (1.1) with $f(\vec{r})=1$ and $g(\bar{r})=y-k^{2}$. After the coordinate scaling described above and introducing $\tau=-t$ from Equation (2.6), the equation we consider is

$$
\nabla^{2} \psi-\frac{\partial^{2} \psi}{\partial \tau^{2}}+\lambda^{2}\left(y-k^{2}\right) \psi=0
$$

We assume an asymptotic solution of the form

$$
\psi(\bar{r}, \tau)-\int d \bar{p} d \omega \exp \{i \lambda(\bar{r} \cdot \bar{p}+\omega \tau-S(\bar{p}, \omega))\} A(\bar{r}, \tau, \bar{p}, \omega, \lambda)=0\left(\lambda^{-\infty}\right),
$$

with $A(\bar{r}, \tau, \bar{p}, \omega, \lambda)=1$ at the emitter. Proceeding through the algorithm, we obtain the Hamiltonian

$$
H=\bar{p} \cdot \bar{p}-\omega^{2}+y-k^{2}
$$

and using Hamilton's Equations we find the maps

$$
\begin{array}{ll}
x=2 \mu \gamma \sin \theta & p_{x}=\mu \sin \theta \\
y=-\gamma^{2}+2 \mu \gamma \cos \theta & p_{y}=-\gamma+\mu \cos \theta \\
\tau=-2 \Omega \gamma & \omega=\Omega
\end{array}
$$

In these equations $\gamma$ is the ray path parameter, $\theta$ is taken with respect to the positive (vertical) y axis, $\Omega$ is the initial frequency and $\mu=\left(\Omega^{2}+k^{2}\right)^{1 / 2}$, from Equation (3.6). Combining these maps leads to the Lagrange manifold

$$
\begin{aligned}
& y=\omega^{2}+k^{2}-p_{x}{ }^{2}-p_{y}^{2}=\frac{\partial S}{\partial p_{y}} \\
& x=2 p_{x}\left(\omega^{2}+k^{2}-p_{x}^{2}\right)^{1 / 2}-2 p_{x} p_{y}=\frac{\partial S}{\partial p_{x}} \\
& \tau=2 \omega p_{y}-2 \omega\left(\omega^{2}+k^{2}-p_{x}^{2}\right)^{1 / 2}=\frac{\partial S}{\partial \omega}
\end{aligned}
$$

and the phase

$$
\phi(\bar{r}, \tau, \bar{p}, \omega)=\bar{r} \cdot \bar{p}+\omega \tau+\frac{1}{3} p_{y}^{3}+p_{y}\left(p_{x}{ }^{2}-\omega^{2}-k^{2}\right)+\frac{2}{3}\left(\omega^{2}+k^{2}-p_{x}\right)^{3 / 2} .
$$

Equating the Hessian determinant of the phase to zero determines the equation of the caustic in $(\bar{p}, \omega)$ - space, cf. Equation (3.16) 
$\left(2 p_{y}^{2}+\omega^{2}-p_{x}^{2}\right)\left(\omega^{2}+k^{2}-p_{x}^{2}\right)^{1 / 2}+p_{y}^{2}\left(\omega^{2}-p_{x}^{2}\right)\left(\omega^{2}+k^{2}-p_{x}^{2}\right)^{-1 / 2}+p_{y}\left(3 p_{x}^{2}-3 \omega^{2}-p_{y}^{2}-k^{2}\right)=0$.

Those real $(\bar{p}, \omega)$ satisfying Equation (4.8) project the caustic onto configuration space through the Lagrange manifold. For definiteness, let $\Omega=3$ and $k=4$, thus $\mu=5$. Then one such $(\bar{p}, \omega)$ set is $\left(p_{x}, p_{y}, \omega\right)=(4.33,-3.9,3$.$) , leading to (x, y, \tau)=$ $(55.4,-8.96,-38.4)$ and hence the space time point $(x, y, t)=(55.4,-8.96,38.4)$. For this $(\bar{p}, \omega)$ set we $f$ ind from the maps above $(\gamma, \theta, \Omega)=\left(6.4,60^{\circ}, 3.\right)$. We note that at this $(\gamma, \theta, \Omega)$

$$
\operatorname{det}\left(\frac{\partial(x, y, \tau)}{\partial(\gamma, \partial, \lambda)}\right)=0
$$

confirming the level-correspondence between the parametrizations at the field point. From the algorithm in the Appendix, we find $n=3$. For computational simplicity, we expand the phase only to third order in Equation (3.24) and, after some algebra, obtain the coordinate transformation

$$
\begin{aligned}
& \beta_{1}=\left(6.38-.02 \alpha_{1}-1.08 \alpha_{3}-1.99 \alpha_{2}\right)^{1 / 2} \alpha_{1}+66 \alpha_{3}{ }^{2}\left(6.38-.02 \alpha_{1}-1.08 \alpha_{3}-1.99 \alpha_{2}\right)^{-1 / 2} \\
& \beta_{2}=\left(13.65+1.88 \alpha_{2}+4.77 \alpha_{3}-3.25 \alpha_{1}\right)^{1 / 2} \alpha_{2}+1.45 \alpha_{3}{ }^{2}\left(13.65+1.88 \alpha_{2}+4.77 \alpha_{3}-3.25 \alpha_{1}\right)^{-1 / 2}
\end{aligned}
$$

$\partial_{3}=\left\{-\alpha_{3}{ }^{3}\left(.52+.436 \alpha_{1}\left(6.38-.02 \alpha_{2}-1.08 \alpha_{3}-1.99 \alpha_{2}\right)^{-1}-2.1 \alpha_{3}\left(13.65+1.88 \alpha_{2}+4.77 \alpha_{3}-3.25 \alpha_{1}\right)^{-1}\right\}^{1 / 3}\right.$

which carries $\phi(\bar{r}, \tau, \bar{p}, \omega)$ at $(55.4,-8.96,-38.4,4.33,-3.9,3$.$) to$

$$
\tilde{\bar{\phi}}\left(55.4,-8.96,-38.4, \beta_{1}, \beta_{2}, \beta_{3}\right)=110.4+\beta_{1}^{2}-\beta_{2}^{2}+\beta_{3}^{3} \text {. }
$$

Under this coordinate transformation the field at $(x, y, t)=(55.4,-8.96,38.4)$ is represented by

$\psi(55.4,-8.96,-38.4)=\int \mathrm{d} \bar{B} \mathrm{~A}(55.4,-8.96,-38.4, \bar{\beta}, \lambda) \exp \left\{i \lambda\left(110.4+B_{1}{ }^{2}-B_{2}{ }^{2}+B_{3}{ }^{3}\right)\right\}$

The first two terms in the asymptotic series of Equation (4.9) are

$$
\begin{aligned}
\psi(55.4,-8.96,-38.4) \simeq & .46 \lambda^{-4 / 3} \exp \{i \lambda(110.4 \pi)\} \Gamma\left(\frac{1}{3}\right) \cos (\pi / 6)+ \\
& .11 \lambda^{-5 / 3} \exp \{1 \lambda(110.4 \pi)\} \Gamma(2 / 3) \sin (\pi / 3) .
\end{aligned}
$$

\section{VECTOR FIELDS}

The same algorithm applies to vector (electric or magnetic) field propagation. Using the electric field, $E$, as an example, the vector field equation analogous to Equation (1.2) is

$$
\nabla^{2} \bar{E}(\bar{r}, \tau)-f(\bar{r}) \frac{\partial^{2} \bar{E}}{\partial \tau^{2}}-\lambda^{2} g(\bar{r}) \bar{E}=0
$$

where $\bar{E}(\bar{r}, \tau)$ is a column vector. Analogous to Equation (2.5) we assume an asymptotic solution of the form

$$
\bar{E}(\bar{r}, \tau)-\sqrt{E}(\bar{r}, \tau, \bar{p}, \omega, \lambda) \exp \{i \lambda(\bar{r} \cdot \bar{p}+\omega \tau-S(\bar{p}, \omega))\} d \bar{p} d \omega=0\left(\lambda^{-\infty}\right)
$$


where

$$
\bar{E}(\bar{r}, \tau, \bar{p}, \omega, \lambda) \simeq \sum_{k=0} \bar{E}_{k}(\bar{r}, \tau, \bar{p}, \omega, \lambda)(i \lambda)^{-k}
$$

Proceeding through the algorithm, we find a transport equation results if we require in a neighborhood of the Lagrange manifold that

$$
-\left(\nabla_{q} \cdot \bar{D}\right) \bar{E}+\bar{D} \nabla_{q} \cdot \bar{E}+2 \bar{p}(\nabla \cdot E)-2 \omega f(\bar{r}) \frac{3 \bar{E}}{j \tau}+\frac{1}{i, i}\left[\nabla_{r}^{2} \bar{E}-f(\bar{r}) \frac{3^{2} E}{\partial \tau^{2}}\right]=0
$$

where the symbols follow from the scalar field treatment. Then introducing the flow

$$
\begin{array}{ll}
\frac{d \bar{r}}{d \gamma}=2 \bar{p} & \frac{d \bar{p}}{d \gamma}=-\bar{D}_{r} \\
\frac{d \tau}{d \gamma}=-2 \omega f(\bar{r}) & \frac{d \omega}{d \gamma}=-D_{\tau},
\end{array}
$$

leads to the transport equation

$$
\frac{d \bar{E}}{d \gamma}-\left(\nabla_{q} \cdot \bar{D}\right) \bar{E}+\frac{1}{i \lambda}\left[\nabla^{2} \bar{E}-f(\bar{r}) \frac{\partial^{2} E}{\partial \tau^{2}}\right]=0 .
$$

As with the field away from the caustic [7], the zeroth-order approximation to the time-averaged Poynting vector (power density) $S$ on the caustic

$$
\bar{S}=\operatorname{Re}\left(\bar{E}_{\mathbf{x}} \bar{F}\right)=\left(\frac{\varepsilon_{0}}{\mu_{0}}\right)^{1 / 2} E_{0}{ }^{2-},
$$

where $\varepsilon_{0}$ and $\mu_{0}$ are the permittivity and permeability, respectively, of vacuum proceeds from the zeroth-order term of Equation (5.3), i.e.,

$$
-\left(\nabla_{q} \cdot \bar{D}\right) \bar{E}_{0}-\bar{D}\left(\nabla_{q} \cdot \bar{E}_{0}\right)+2 \bar{p}\left(\nabla \cdot \bar{E}_{0}\right)-2 \omega f(\bar{r}) \frac{\partial \bar{E}_{o}}{\partial \tau}=0 .
$$

Scalar multiplication of Equation (5.5) by $\bar{E}_{0} *$ and similarly multiplying the complex conjugate of (5.5)

$$
-\left(\nabla_{q} \cdot \bar{D}\right) \bar{E}_{0}^{*}-D\left(\nabla_{q} \cdot \bar{E}_{0}^{*}\right)+2 \bar{p}\left(\nabla \cdot \bar{E}_{0}^{*}\right)-2 \omega f(\bar{r}) \frac{\partial \bar{E}_{0}^{*}}{\partial \tau}=0
$$

by $\bar{E}_{0}$ and introducing the flow above leads to

$$
\begin{aligned}
& \frac{d E_{o}^{2}}{d \gamma}-2 E_{o}^{2}\left(\nabla_{q} \cdot \bar{D}\right)=0 \\
& E_{o}^{2}(\gamma)=E_{o}^{2}(\gamma=0) \exp \left\{2 \int\left(\nabla_{q} \cdot \bar{D}\right) d_{\gamma}\right\} .
\end{aligned}
$$

A similar consideration leads to a transport equation for the polarization

$$
\bar{P}=\frac{\bar{E}_{0}}{\left(\bar{E}_{0} \cdot \bar{E}_{0}{ }^{*}\right)^{1 / 2}} \text {. }
$$

First, following Felsen and Marcuvitz [7], we differentiate Equation (5.9) to obtain

$$
\frac{d P}{d \gamma}=\frac{1}{E_{0}} \frac{d \bar{E}_{o}}{d \gamma}-\frac{\bar{E}_{o}}{E_{0}^{2}} \frac{d E_{0}}{d \gamma} .
$$


Then combining Equation (5.7) with the first term of Equation (5.5) and noting that the remaining three terms

leads to

$$
-\bar{D}\left(\nabla_{q} \cdot \bar{E}_{o}\right)+2 \bar{p}\left(\nabla_{q} \cdot \bar{E}_{o}\right)-2 \omega f(r) \frac{\partial \bar{E}_{o}}{\partial \tau}=\frac{d \bar{E}_{o}}{d \gamma}
$$

$$
-\bar{E}_{o}\left(\frac{1}{E_{0}} \frac{d E_{o}}{d \gamma}\right)+\frac{d E_{o}}{d \gamma}=0 .
$$

Dividing Equation (5.11) by $\mathrm{E}_{\mathrm{o}}$ and comparing with Equation (5.10) obtains the interesting result that on the flow defined above

$$
\frac{d P}{d \gamma}=0
$$

the polarization is a constant, extending the off-caustic result of Felsen and Marcuvitz.

While this development has been concerned with dispersive waves, a parallel development obtains analogous results in the non-dispersive case.

APPENDIX

Consider the phase function $\phi(\bar{r}, \tau, \bar{p}, \omega)$ such that

$$
\left(\hat{p} \nabla_{p}+\hat{\omega} \frac{\partial}{\partial \omega}\right) \phi\left(\bar{r}_{o}, \tau, \bar{p}_{o}, \omega_{o}\right)=0
$$

and the Hessian of the phase at $\left(\bar{r}_{0}, \tau_{0}, \bar{p}_{0}, \omega_{0}\right)$ has at least two non-zero eigenvalues. If the Hessian has no zero eigenvalues the canonical form for the phase is

$$
\tilde{\bar{\phi}}\left(\overline{\mathbf{r}}_{0}, \tau_{0}, \bar{B}\right)=\phi\left(\bar{r}_{0}, \tau_{0}, \bar{p}_{0}, \omega_{0}\right) \pm B_{1}{ }^{2} \pm B_{2}{ }^{2} \pm B_{3}{ }^{2} \pm B_{4}{ }^{2} \text {, }
$$

where the signs of the $B_{1}$ are determined by the corresponding eigenvalues. If the Hessian has one zero eigenvalue the canonical form for the phase is

$$
\tilde{\bar{\phi}}\left(\bar{r}_{0}, \tau_{0}, \bar{B}\right)=\phi\left(\bar{r}_{0}, \tau_{0}, \bar{p}_{0}, \omega_{0}\right) \pm \beta_{1}{ }^{2} \pm \beta_{2}{ }^{2} \pm \beta_{3}{ }^{2} \pm B_{4}{ }^{n} .
$$

To determine the value of $n$ we form

$$
F(\mu)=\left(\bar{r}_{0}, \tau_{0}, \bar{p}_{0}+\mu \bar{e}_{p}, \omega_{0}+\mu e_{\omega}\right)
$$

where $\left(\bar{e}_{p}, e_{\omega}\right)$ are the components of the eigenvector corresponding to the zero eigenvalue. The exponent of the first non-vanishing term in the Taylor series determines the value of $n$. The sign of $B_{4}$ is the sign of the Taylor coefficient.

If the Hessian has two zero eigenvalues, the canonical form for the phase is

$$
\tilde{\bar{\phi}}\left(\bar{r}_{0}, \tau_{0}, \bar{\beta}\right)=\phi\left(\bar{r}_{0}, \tau_{0}, \bar{p}_{0}, \omega_{0}\right) \pm \beta_{1}{ }^{2} \pm \beta_{2}{ }^{2}+U\left(\beta_{3}, \beta_{4}\right) \text {, }
$$

where $U\left(B_{3}, B_{4}\right)$ is a Thom umbilic. To specify the particular umbilic, the phase is first expanded and regrouped as in Equation (3.29). The appropriate umbilic is determined by considering the homogeneous cubic

$$
\kappa_{30} \xi_{3}^{3}+\kappa_{21} \xi_{33}^{3} \xi_{4}+\kappa_{12} \xi_{3} \xi_{4}^{2}+\kappa_{03} \xi_{4}^{3} \text {. }
$$


If $\kappa_{30} \neq 0$, equating the cubic terms to zero and dividing by $\xi_{4}{ }^{3}$ obtains

$$
\kappa_{30} u^{3}+\kappa_{21} u^{2}+\kappa_{12} u+\kappa_{03}=0 \text {, }
$$

where $u=\xi_{3} / \xi_{4}$. (If $\kappa_{30}$ and $\kappa_{03} \neq 0$, interchanging $\xi_{3}$ and $\xi_{4}$ yields an analogous cubic.) The cubic may have four possible root combinations, each corresponding to a specific canonical form [19].

(a) three real equal roots $\beta^{3}$ (fold)

(b) three real unequal roots $B_{3}{ }^{3}-B_{3} B_{4}^{2} \quad$ (elliptic umbilic)

(c) three real roots (two equal)

$\mathrm{B}_{3}^{2} \mathrm{~B}_{4}+\mathrm{B}_{4}^{4}$ (parabolic umbilic)

(d) one real root, one complex $\mathrm{B}_{3}{ }^{3}+\mathrm{B}_{4} \mathrm{~B}_{3}^{2}$ (hyperbolic umbilic). (or $B_{3}{ }^{3}+\mathrm{B}_{4}^{3}$ )

If $\kappa_{30}=\kappa_{03}=0$ and both $\kappa_{21}, \kappa_{12} \neq 0$, the corresponding form is the parabolic umbilic. If $\kappa_{30}=\kappa_{03}=0$ and one of $\kappa_{21}$ or $\kappa_{12}=0$, there is no corresponding canonical form. (The case corresponding to three real equal roots represents an atypical symmetry and is not considered here.) If the Hessian matrix of the phase has more than two zero eigenvalues, this algorithm does not apply.

ACKNOWLEDGEMENTS: Helpful discussions with R. Folk, W. J. Guy and A. Novaco and the continuing pedgagogy of $R$. Wells are gratefully acknowledged. Elizabeth Bullock typed the manuscript. This work was partially supported by NSF grant DMS- 8409392 .

\section{REFERENCES}

1. KELlER, J. B. in CALCULUS OF VARIATIONS AND ITS APPLICATIONS (McGraw-Hill, New York, 1958).

2. WEINBERG, S. Eikonal Method in Magnetohydrodynamics, Phys. Rev., 126 (1962) 1899-1909.

3. STIX, T. H. THE THEORY OF PLASMA WAVES (McGraw-Hill, New York, 1962).

4. GRANOFF, B. and LEWIS, R. M. Asymptotic Solution of Initial Boundary-Value Problems for Hyperbolic Systems, Phil. Trans. Royal Soc., Ser. A., 262 (1967) 387-411.

5. BRANBILLA, M. and CARDINALI, A. Eikonal Description of High Frequency waves in Toroidal Plasmas, Plasma Physics, 26 (1982) 1187-1218.

6. HAZAK, G., BERNSTEIN, I. and SMITH, T. M. Integral Representation for Geometric Optics Solutions, Phys. Fluids, 26 (1983) 684-688.

7. FELSEN, L. and MARCUVITZ, N. RADIATION AND SCATTERING OF WAVES (Prentice Hall, Englewood Cliffs, New Jersey, 1973).

8. ZAUDERER, E. PARTIAL DIFFERENTIAL EQUATIONS OF APPLIED MATHEMATICS (John Wiley $\&$ Sons, New York, 1984).

9. MASLOV, V. P. THEORIE DES PERTURBATIONES ET METHODES ASYMPTOTIQUES (Dunod, Gauthier-Villars, Paris, 1972).

10. ARNOLD, V. I. Characteristic Class Entering in Quantization Conditions, Funct. Ana1. App 1., 1 (1967) 1-13. 
11. GORMAN, A. D., WELlS, R. and FlEMing, G. N. Wave Propagation and Thom's Theorem, J. Phys. A., 14 (1981) 1519-1531.

12. BERRY, M. V. Waves and Thom's Theorem, Adv. Phys., 25 (1976) 1-25.

13. ARNOLD, V. I. Singularities of Systems of Rays, Russ. Math. Surveys, 38 (1983) $87-176$.

14. GROMOLL, D. and MEYER, W. On Differentiable Functions with Isolated Critical Points, Topology, $\underline{8}$ (1969) 361-370.

15. POSTON, T. and STEWART, I. TAYLOR EXPANSIONS AND CATASTROPHES (PItman, London, 1976).

16. GILMORE, R. CATASTROPHE THEORY FOR SCIENTISTS AND ENGINEERS (John Wiley \& Sons, New York, 1981).

17. URSELL, F. Integrals with a Large Parameter: A Double Complex Integral with Four Nearly Coincident Saddle Points, Math. Proc. Camb. Phil. Soc., 87 (1980) 249-274.

18. GORMAN, A. D. and WELLS, R. On the Asymptotic Expansion of Certain Canonical Integrals, J. Math. Anal. Applics., 102 (1984) 566-584.

19. POSTON, T. and STEWART, I. CATASTROPHE THEORY AND ITS APPLICATIONS (PItman, London, 1978). 


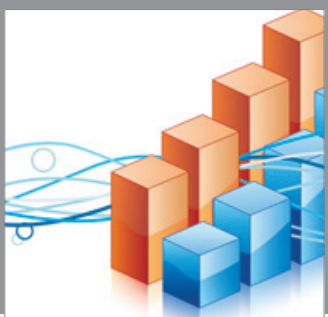

Advances in

Operations Research

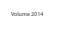

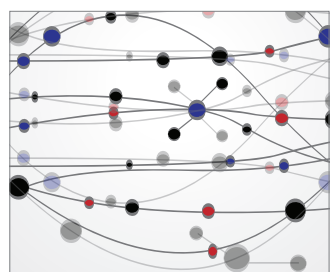

\section{The Scientific} World Journal
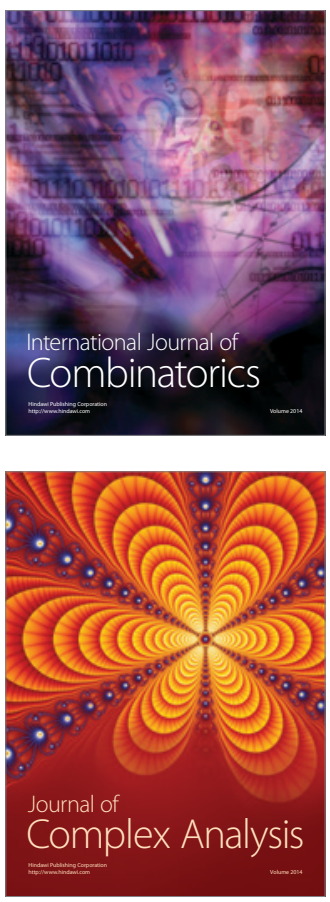

International Journal of

Mathematics and

Mathematical

Sciences
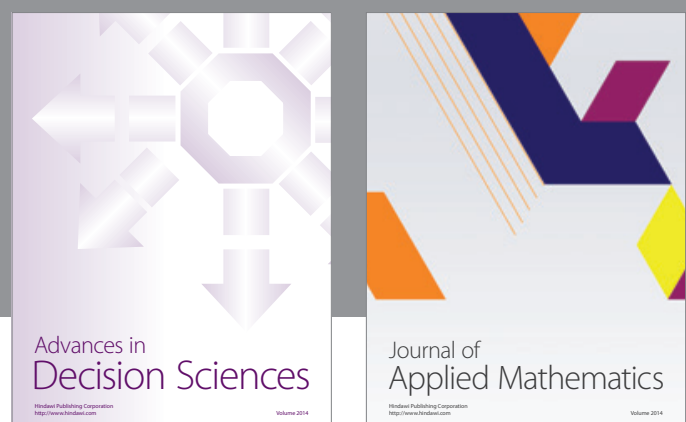

Journal of

Applied Mathematics
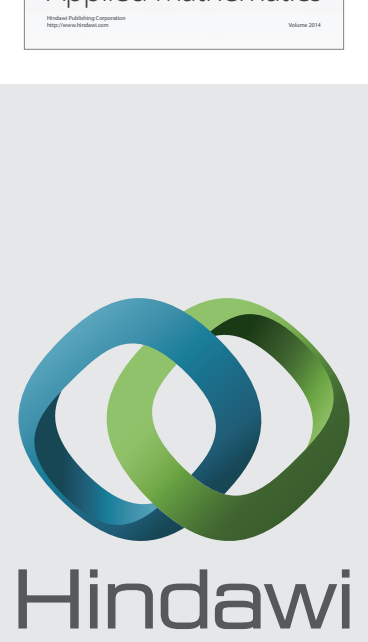

Submit your manuscripts at http://www.hindawi.com
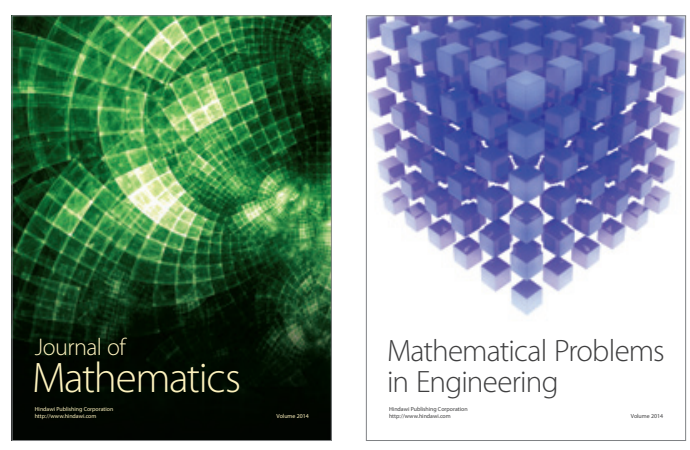

Mathematical Problems in Engineering
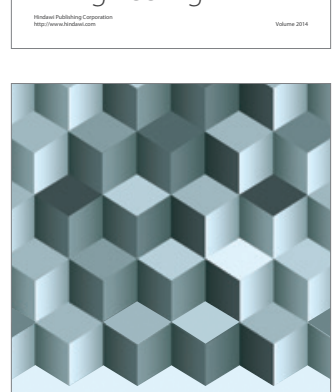

Journal of

Function Spaces
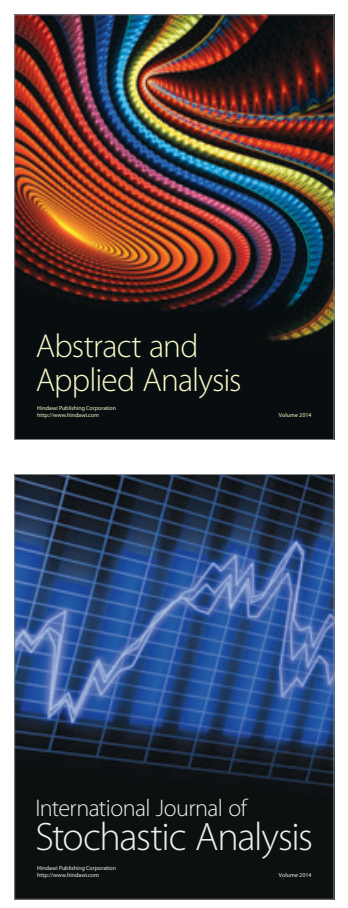

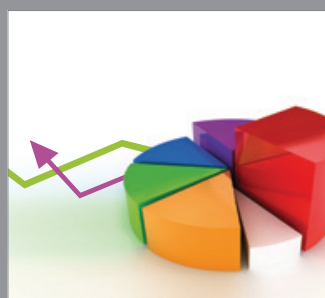

ournal of

Probability and Statistics

Promensencen
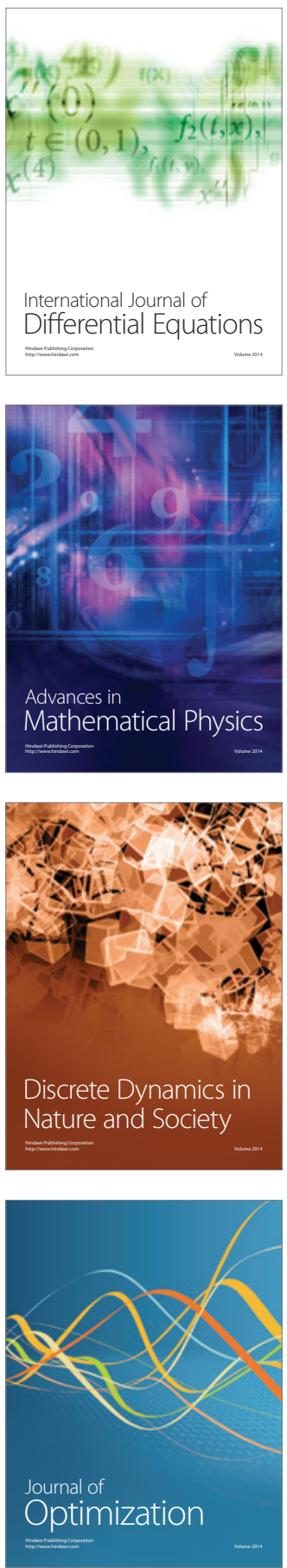